\title{
Nile Red-Adsorbed Gold Nanoparticles for Selective Determination of Thiols Based on Energy Transfer and Aggregation
}

\author{
Shih-J u Chen and Huan-Tsung Chang* \\ Department of Chemistry, National Taiwan University, Roosevelt Road, Section 4, Taipei 106, Taiwan, R.O.C.
}

For the first time, an aqueous solution of 32-nm gold nanoparticles (GNPs), to which Nile red (NR) has been noncovalently adsorbed, has been used for sensing thiols. The as-prepared NRGNPs fluoresce weakly as a result of fluorescence resonance energy transfer between NR and the GNPs. The fluorescence of a solution containing NRGNPs at pH 4.0 increases upon the addition of thiols, but not when amines, acids, alcohols, bovine serum albumin, or hemoglobin are added. This phenomenon allows for the selective determination of thiols such as cysteamine and homocysteine, which have limits of detection of $\mathbf{1 0 . 2}$ and $\mathbf{1 0 . 9} \mathrm{nM}$, respectively, at a signal-to-noise ratio of 3. Interestingly, we have found that the excitation $\left(\lambda_{\mathrm{ex}}=480 \mathrm{~nm}\right)$, emission $\left(\lambda_{\mathrm{em}}=610 \mathrm{~nm}\right)$, and mass spectra $(\mathrm{m} / \mathrm{z} 282)$ of the substance that desorbs from the GNPs in the presence of thiols are different from those of NR $\left(\lambda_{\mathrm{ex}}=580 \mathrm{~nm} ; \lambda_{\mathrm{em}}=652 \mathrm{~nm} ; \mathrm{m} / \mathrm{z} 318\right)$, which indicates that a new product forms. When simultaneously conducting fluorescence and colorimetric assays, the selectivity of this approach further improves because at pH 4.0, the color of the NRGNPs does not change in the presence of negatively charged thiols, (e.g., N-(2-mercaptopropionyl)glycine), but changes from maroon to purple and lavender in the presence of neutral thiols (e.g., 3-mercapto-1,2-propanediol) and positively charged thiols (e.g., cysteamine), respectively, as a result of aggregation. This feature allows the types of thiols to be determined at concentrations $>1.0$ and $0.1 \mu \mathrm{M}$ by the naked eye and by UV-vis absorption, respectively. Depending on the rate at which the NRGNP color changes, reduced glutathione (slow) is readily distinguishable from oxidized glutathione (no aggregation and no displacement) and from cysteine and homocysteine (fast).

Nanoparticles (NPs) are becoming increasingly attractive materials for biosensors because of their dimensional similarities with biomacromolecules and because they have size-dependent optical and electronic properties. ${ }^{1-4} \mathrm{~A}$ feature that makes them

* To whom correspondence should be addressed. Phone and Fax: 011-8862-23621963. E-mail: changht@ntu.edu.tw.

(1) Bruchez, M ., J r.; M oronne, M .; Gin, P.; Weiss, S.; Alivisatos, A. P. Science 1998, 281, 2013-2016.

(2) Chan, W. C. W.; Nie, S. Science 1998, 281, 2016-2018.

(3) Niemeyer, C. M. Angew. Chem. Int. Ed. 2001, 40, 4128-4158.

(4) Veiseh, M.; Zareie, M. H.; Zhang, M. Langmuir 2002, 18, 6671-6678. particularly appealing is that color changes induced by association of gold nanoparticles (GNPs) provide the basis for a simple, yet highly selective, approach to the analysis of a wide number of analytes. $^{5-12}$ Oligonucleotide-modified GN Ps have been prepared and tested for sensing target DNA sequences through hybridization with their complementary oligonucleotide strands. ${ }^{6-8}$ In addition, carbohydrate-functionalized GN Ps, 9,10 antigen-modified GNPs, ${ }^{11}$ and [15]crown-5-functionalized GN Ps ${ }^{12}$ have been used for the analyses of lectins, antibodies, and metal ions, respectively.

In addition, homogeneous assays based on fluorescence resonance energy transfer (FRET) using NPs have great potential for applications in diagnostics. ${ }^{13-15}$ Depending on their optical properties, NPs can act as acceptor (quencher) or donor units. GNPs possessing high extinction coefficients $\left(\sim 8 \times 10^{8} \mathrm{~cm}^{-1}\right.$ $\mathrm{mol}^{-1} \mathrm{~L}$ for $13-\mathrm{nm}$ GNPs at $\left.520 \mathrm{~nm}\right)^{16}$ in the ultraviolet and visible regions are efficient quenchers for most fluorophores. For example, a single-stranded DNA covalently linked with a fluorophore and 1.4-nm GNP has been proposed for detecting a single matched DNA on the basis of quenching of fluorescence by the metal that results from both nonradiative energy transfer from the dye to the metal and from collision of the dye against the gold surface (dynamic quenching). ${ }^{17}$ Alternatively, targeting DN A sequences has been demonstrated using oligonucleotides labeled with a thiol at one end and a dye (e.g., fluorescein or tetramethylrhodamine) at the other..$^{13}$

(5) Nath, N.; Chilkoti, A. Anal. Chem. 2002, 74, 504-509.

(6) Elghanian, R.; Storhoff, J. J.; M usic, R. C.; Letsinger, R. L.; M irkin, C. A. Science 1997, 277, 1078-1081.

(7) Storhoff, J. J.; Elghanian, R.; M ucic, R. C.; M irkin, C. A.; Letsinger, R. L. J. Am. Chem. Soc. 1998, 120, 1959-1964.

(8) Nam, J.-M .; Park, S.J.; M irkin C. A. J. Am. Chem. Soc. 2002, 124, 38203821.

(9) Otsuka, H.; Akiyama, Y.; Nagasaki, Y.; Kataoka, K. J. Am. Chem. Soc. 2001, $123,8226-8230$

(10) Lin, C.-C.; Yeh, Y.-C.; Yang, C.-Y.; Chen, C.-L.; Chen, G.-F.; Chen, C.-C.; Wu, Y.-C. J. Am. Chem. Soc. 2002, 124, 3508-3509.

(11) Thanh, N. T. K.; Rosenzweig, Z. Anal. Chem. 2002, 74, 1624-1628.

(12) Lin, S.-Y.; Liu, S.-W.; Lin, C.-M .; Chen, C.-H. Anal. Chem. 2002, 74, 330335.

(13) M axwell, D. J.; Taylor, J. R.; Nie, S. J. Am. Chem. Soc. 2002, 124, $9606-$ 9612.

(14) Kürner, J. M .; Wolfbeis, O. S.; Klimant, I. Anal. Chem. 2002, 74, 21512156.

(15) Wang, S.; M amedova, N.; Kotov, N. A.; Chen, W.; Studer, J N Nano Lett. 2002, $2,817-822$.

(16) Link, S.; El-Sayed, M. A. J. Phys. Chem. B 1999, 103, 8410-8426.

(17) Dubertret, B.; Calame, M .; Libchaber, A. J. Nat. Biotech. 2001, 19, 365370.

Analytical Chemistry, Vol. 76, No. 13, July 1, 2004

3727 


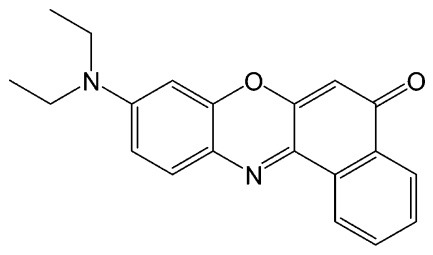

Figure 1. The chemical structure of NR.

A number of thiols, including some aminothiols, are important markers for diagnosing inherited and acquired metabolic disturbances and diseases. ${ }^{18-20}$ Cysteine deficiency is involved in many syndromes, such as slower growth, edema, liver damage, and skin lesions. ${ }^{21} \mathrm{M}$ onitoring total plasma homocysteine levels is important because even mild hyperhomocysteinemia has been found to be an independent risk factor for premature occlusive disease in the coronary, cerebral, and peripheral arteries, and for venous thrombosis. ${ }^{22,23}$ The majority of the reported methods for analysis of aminothiols are based on redox chemistry or derivatization with chromophores or fluorophores. ${ }^{24-26}$ For example, the analysis of aminothiols labeled with 5-(bromomethyl)fluorescein or 6-iodoacetamidofluorescein has been performed separately by capillary electrophoresis in conjunction with laser-induced fluorescence ${ }^{19,20}$ with the detection limits in the range of $0.01-1 \mu \mathrm{M}$. Recently, a very selective fluorescence method has been demonstrated for determining the levels of cysteine and homocysteine using a xanthene dye, with a linear range of their physicological levels (healthy plasma total homocystein concentrations are $\sim 12 \mu \mathrm{M}$ ). ${ }^{27}$

Unlike those studies, herein we report the sensing of thiols using Nile red (NR)-adsorbed GNPs (NRGNPs) based on FRET, aggregation, or both. Nile red (phenoxazine dye 9-diethylamino$5 \mathrm{H}$-benzo[ $\alpha]$ phenoxazin-5-one), which is presented in Figure 1 , is a hydrophobic, highly fluorescent, solvatochromic dye that undergoes intensity or wavelength shifts upon changes in the polarity of its environment. ${ }^{28-30}$ These distinct characteristics make micro- and nanostructured materials containing NR good sensing materials for air monitoring and odor discrimination. ${ }^{31-34}$ NRGNPS

(18) M udd, S. H.; Levy, H. L.; Skovby, F. In The M etabolic and M olecular Basis of Inherited Disease; Scriver, C. R., Beaudet, A. L., Sly, W. S., Valle, D., Eds.; M cGraw-Hill, New York, 1995.

(19) Lochman, P.; Adam, T.; Friedecký, D.; Hlídková, E.; Šopková, Z. Electrophoresis 2003, 24, 1200-1207.

(20) Caussé, E.; Malatray, P.; Calaf, R.; Charpiot, P.; Candito, M.; Bayle, C.; Valdiguié, P.; Salvayre, R.; Couderc, F. Electrophoresis 2000, 21, 20742079.

(21) Shahrokhian, S. Anal. Chem. 2001, 73, 5972-5978.

(22) Refsum, H.; Ueland, P. M.; Nygard, O.; Vollset, S. E. Annu. Rev. M ed. 1998, 49, 31-62.

(23) Cavalca, V.; Cighetti, G.; Bamonti, F.; Loaldi, A.; Bortone, L.; N ovembrino, C.; De Franceschi, M.; Belardinelli, R.; Guazzi, M. D. Clin. Chem. 2001, $47,887-892$

(24) Nekrassova, O.; Lawrence, N. S.; Compton, R. G. Talanta 2003, 60, 10851095.

(25) Chou, S.-T.; Ko, L.-E.; Yang, C.-S. Anal. Chim. Acta 2001, 429, 331-336.

(26) Houze, P.; Gamra, S.; M adelaine, I.; B ousquet, B.; Gourmel, B. J. Clin. Lab. Anal. 2001, 15, 144-153.

(27) Rusin, O.; St. Luce, N. N.; Agbaria, R. A.; Escobedo, J. O.; Jiang, S.; Warner, I. M.; Dawan, F. B.; Lian, K.; Strongin, R. M . J. Am. Chem. Soc. 2004, 126, 438-439.

(28) Ghoneim, N. Spectrochim. Acta A 2000, 56, 1003-1010.

(29) Dutta, A. K.; Kamada, K.; Ohta, K. J. Photochem. Photobiol. A 1996, 93, $57-64$.

(30) Hungerford, G.; Ferreira, J. A. J. Luminescence 2001, 93, 155-165.

(31) Levitsky, I. A.; Krivoshlykov S. G.; Grate, J. W. J. Phys. Chem. B 2001, 105, 8468-8473. fluoresce weakly because FRET takes place between NR and the GNPs. The fluorescence increases as a result of a product desorbing from the GNP surface upon the addition of thiols that possess strong $\mathrm{Au}-\mathrm{S}$ bonding interactions with GNPs. By conducting a colorimetric assay using NRGN Ps, positively charged and neutral thiols that at pH 4.0 induce color changes as a result of the aggregation of the particles can be discriminated from negatively charged thiols. The depiction in Figure 2 demonstrates the mechanisms of the assays we present in this report. A dual assay based on FRET (increases in fluorescence at $610 \mathrm{~nm}$ ) and aggregation (changes in color) is useful for detecting neutral and positively charged thiols, while only the FRET mechanism can be applied to negatively charged thiols. The color changes are used to further distinguish positively charged thiols (lavender) from neutral thiols (purple).

\section{EXPERIMENTAL SECTION}

Chemicals. Sodium tetrachloroaurate(III) dihydrate, bovine serum albumin (BSA), cysteamine, sodium cyanide, and D-(-)penicillamine were obtained from Sigma (St. Louis, M O). Acetic acid, acetonitrile, cysteine, ethanol, formic acid, glutathione, homocysteine, hydrogen chloride, 2-propanol, N-(2-mercaptopropionyl)glycine, 2-mercaptoethanol (2-M E), 3-mercapto-1,2-propanediol, 3-mercaptopropionic acid, propylamine, sodium mercaptoacetate, sodium hydroxide, and trisodium citrate were purchased from Aldrich (M ilwaukee, WI). NR was obtained from Acros (Geel, Belgium). Citrate solutions were adjusted, using either $\mathrm{HCl}$ or $\mathrm{NaOH}$, to values of $\mathrm{pH}$ ranging from 4.0 to 9.0 . Isotonic phosphate-buffered saline (PBS; pH 7.4) was prepared by dissolving $\mathrm{Na}_{2} \mathrm{HPO}_{4} \cdot 12 \mathrm{H}_{2} \mathrm{O}(22.05 \mathrm{~g}), \mathrm{NaH}_{2} \mathrm{PO}_{4} \cdot 2 \mathrm{H}_{2} \mathrm{O}(2.07 \mathrm{~g})$, and $\mathrm{NaCl}(4.5 \mathrm{~g})$ in $\mathrm{H}_{2} \mathrm{O}(1 \mathrm{~L})$.

Synthesis of GNPs; Preparation of 32-nm GNPs. Trisodium citrate $(1 \% 0.5 \mathrm{~mL})$ was added rapidly to an aliquot of $0.01 \%$ $\mathrm{HAuCl}_{4}(50 \mathrm{~mL})$ that was heated under reflux. 35,36 The solution was heated under reflux for an additional $8 \mathrm{~min}$, during which time the color changed to deep red. The solution was set aside to cool to room temperature and was stable for at least 6 months. Please note that we denote the concentration of the as-prepared GNPs to be $1 \times$ (the concentration is $\sim 1.6 \times 10^{11}$ particles $/ \mathrm{mL}=$ $0.27 \mathrm{nM}) .{ }^{37}$

Characterization of GNPs. A double-beam UV-vis spectrophotometer (Cintra 10e) obtained from GBC (Victoria, Australia) was used to measure the absorbance of the GNPs in citrate solutions. For the 32-nm GN Ps, UV -vis absorption measurements (not shown) indicated that the maximum wavelength of the surface plasmon resonance (SPR) was $528 \mathrm{~nm}$, which suggests that the size of these GN Ps is as expected. A fluorometer (AmincoBowman) obtained from ThermoSpectronic (Pittsford, NY) was used to collect the fluorescence spectra of NR and NRGNPs in the presence and absence of thiols. The emission spectra were recorded while irradiating the solution at $543 \mathrm{~nm}$. The NRGNPs in the absence and presence of thiols and BSA were imaged using

(32) M einershagen, J. L.; Bein, T. J. Am. Chem. Soc. 1999, 121, 448-449.

(33) Albert, K. J.; Walt, D. R.; Gill, D. S.; Pearce; T. C. Anal. Chem. 2001, 73, 2501-2508.

(34) Albert, K. J.; Walt, D. R. Anal. Chem. 2003, 75, 4161-4167.

(35) Frens, G. Nature 1973, 241, 20-22.

(36) Grabar, K. C.; Freeman, R. G.; Hommer, M. B.; Natan, M. J. Anal. Chem. 1995, 67, 735-743.

(37) Jana, N. R.; Gearheart, L.; M urphy, C. J. Langmuir 2001, 17, 6782-6786. 


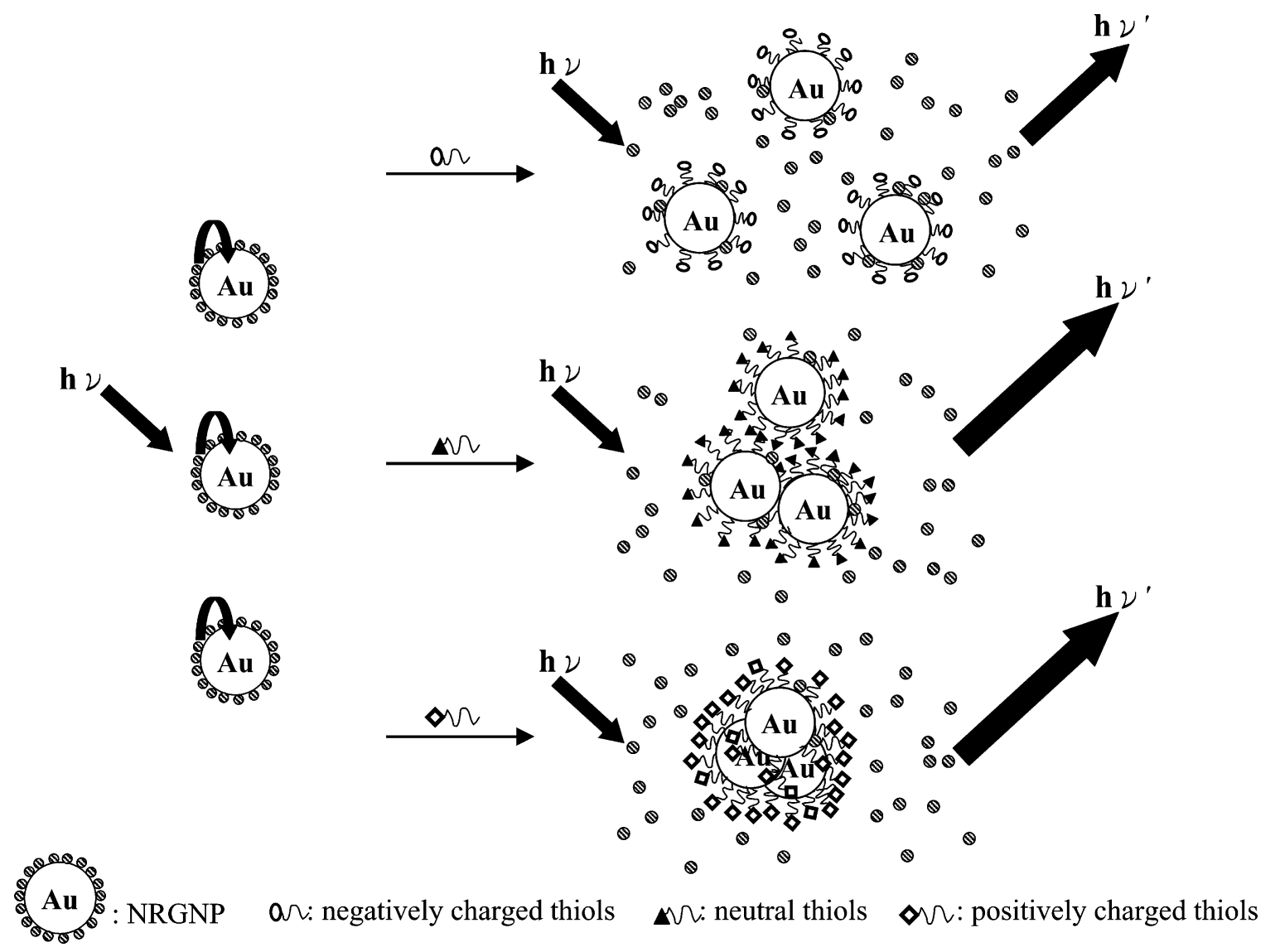

Figure 2. Depiction of the mechanisms of the assays using NRGNPs based on FRET for thiols and FRET/aggregation for neutral and positively charged thiols.

an H7100 TEM from Hitachi (Tokyo, Japan) operating at $75 \mathrm{keV}$. Samples for transmission electron microscopy (TEM) were prepared by placing drops of two $20-\mu \mathrm{L}$ aliquots of the samples onto a holey-carbon-coated copper grid. After deposition, any remaining solution was wicked away, and the grids were then dried for $1 \mathrm{~h}$ at room temperature. The TEM images (not shown) further confirm that the size of the GNPs is $32( \pm 7.0 \%) \mathrm{nm} .{ }^{37}$

Preparation of NRGNPs. A stock solution of NR (1 mM) was prepared in dimethylsulfoxide and diluted with water if necessary. Aliquots of NR solutions $(0.5-50 \mu \mathrm{L})$ were added separately to the $1 \times$ solution of GNPs (4999.5-4950 $\mu \mathrm{L}$ ) such that the final volume of the mixture was $5 \mathrm{~mL}$ and the final concentrations of $\mathrm{NR}$ ranged from 0.1 to $10 \mu \mathrm{M}$. The solutions were equilibrated at ambient temperature and pressure overnight and then subjected to two repeated cycles of centrifugation [at $12000 \mathrm{rpm}$ (relative centrifugal force $=16421 \mathrm{~g}$ ) for $10 \mathrm{~min}$ and washing with citrate buffer $(5 \mathrm{~mL})]$. Finally, the precipitates were resuspended in citrate buffer. We denote the as-prepared solutions as NRGNPs, and for simplicity, their concentration is presented as $1 \times$.

Determination of Thiols. Different thiols were added separately to $1 \times$ NRGNP solutions ( $1 \times$ GNPs and $1 \mu \mathrm{M}$ NR unless otherwise noted) and equilibrated for $10 \mathrm{~min}$. To investigate the selectivity of NRGNPs for thiols, $1.0 \mathrm{mM}$ solutions of acids, amines, alcohols, hemoglobin, and BSA $(0.5 \mathrm{~mL})$ were added separately to $1 \times$ NRGNPs $(4.5 \mathrm{~mL})$ containing 2-ME $(1.25 \mu \mathrm{M})$. After $10 \mathrm{~min}$, both the fluorescence and UV-vis absorptions of the solutions were recorded.
Measuring Molecular Mass. Prior to measuring molecular masses, the solution of NRGNPs was subjected to two repeated cycles of centrifugation (12000 rpm for $10 \mathrm{~min}$ ) and washing with water $(5 \mathrm{~mL})$ to minimize the interference of salt. 2-M E $(10 \mu \mathrm{M}$, $5 \mathrm{~mL}$ ) was added to the precipitate, and the solution was subjected to sonication for $1 \mathrm{~min}$. After $10 \mathrm{~min}$, the solution was centrifuged at $12000 \mathrm{rpm}$ for $10 \mathrm{~min}$, and the supernatant was concentrated $(\sim 50$-fold) by using a rotary evaporator. To conduct mass measurements using a BioTOF electrospray-ionization time-offlight mass spectrometer (Bruker Daltonics, Billerica, USA), the product was dissolved in a $50 \%$ aqueous methanol solution and infused into a sheathless capillary at a flow rate of $80 \mu \mathrm{L} / \mathrm{h}$.

Capillary Electrophoresis. A solution containing the NR product (used for M S measurements) and NR $(1 \mu \mathrm{M})$ was subject to separation by capillary electrophoresis (CE) ${ }^{38} \mathrm{~A} \mathrm{He}-\mathrm{Ne}$ laser ( $543 \mathrm{~nm}$ ) was used in the CE system to excite the analytes; the fluorescence intensity was recorded at wavelengths $>610 \mathrm{~nm}$. The background electrolyte consisted of $5 \% \mathrm{HCOOH}$ and $20 \%$ acetonitrile ( $\left.\mathrm{pH}^{*} 1.9\right)$. The sample was injected hydrodynamically (15-cm height for $2 \mathrm{~s}$ ) and then separated under an applied voltage of $15 \mathrm{kV}$.

\section{RESULTS AND DISSCUSION}

FRET between NR and GNPs. The as-prepared GN Ps having a diameter of $32 \mathrm{~nm}$ were stable in citrate solutions at $\mathrm{pH}>3.5$, mainly because of Coulombic repulsion between the negatively

(38) Chiu, T.-C.; Chang, H.-T. J. Chromatogr., A 2002, 979, 299-306. 


\section{Table 1. Effect of pH on the Fluorescence of Various Solutions}

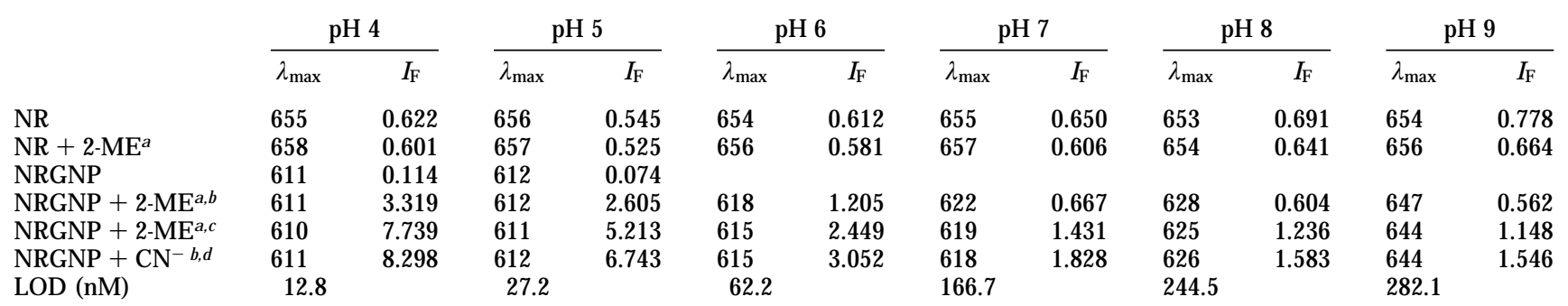

${ }^{a}[2-\mathrm{ME}]=10 \mu \mathrm{M} .{ }^{\mathrm{b}} \mathrm{No}$ centrifugation. ${ }^{\mathrm{c}}$ The supernatants after centrifugation at $12000 \mathrm{rpm}$ for $10 \mathrm{~min} .{ }^{\mathrm{d}}\left[\mathrm{CN}^{-}\right]=20 \mathrm{mM}^{\mathrm{C}}$ Conditions as presented in Figure 3.

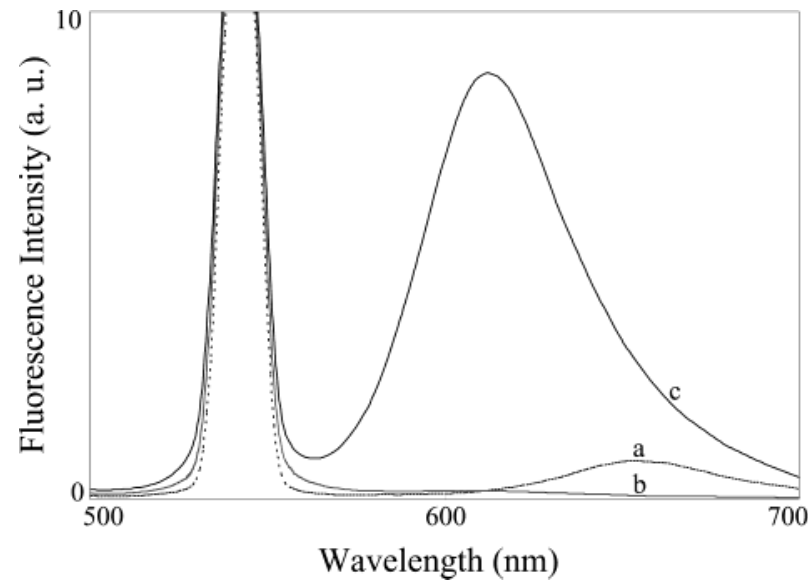

Figure 3. Emission spectra of solutions containing (a) NR and (b) NRGNPs in the absence and (c) the presence of 2-ME $(10 \mu \mathrm{M})$. The concentrations of the GNPs and NR were $1 \times$ and $1.0 \mu \mathrm{M}$, respectively.

charged GNPs capped with citrate ions. The stability of GNPs is similar in the presence of small amounts of NR $(<3.0 \mu \mathrm{M})$, which is evidenced by no change being observed in their SPR band at $528 \mathrm{~nm}$ (not shown). Relative to spectrum a (the emission spectrum for NR) in Figure 3, spectrum b clearly indicates that the fluorescence intensity of NR at $652 \mathrm{~nm}$ decreases dramatically in the presence of the 32-nm GNPs at $\mathrm{pH}$ 4.0. Once the NR molecules noncovalently adsorb onto the GNP surface, FRET occurs between NR and the GNPs. As a result, the fluorescence is quenched, which is a situation similar to the fluorescence quenching of arenes that occurs for gold clusters protected by a monolayer of $\omega$-fluorenyl-alkane-1-thiolate. ${ }^{39} \mathrm{We}$ note that the strong bands appearing near $543 \mathrm{~nm}$ arise from scattering. When 2-ME (10 mM , $2 \mu \mathrm{L}$ ) was added to the NRGNP solution ( $2 \mathrm{~mL}$ ), the color changed to purple, the SPR band shifted from 528 to $538 \mathrm{~nm}$, and the absorption profile became broad, which are all features that suggest that aggregation of the GNPs occurs. At $\mathrm{pH} 4.0$, neutral 2-ME is adsorbed onto the GNP through $\mathrm{Au}-\mathrm{S}$ bonding, which displaces citrate ions, NR, and its product (see below) from the surface. As a result, the negative-charge density of the GNPs decreases and aggregation occurs. Surprisingly, spectrum $c$ in Figure 3 indicates a dramatic increase in the fluorescence intensity and blue shift (from 652 to $610 \mathrm{~nm}$ ) in the presence of 2-M E. After centrifugation, the supernatant fluoresced

(39) Gu, T.; Whitesell, J. K.; Fox, M. A. Chem. M ater. 2003, 15, 1358-1366. even more strongly at $610 \mathrm{~nm}$, which suggests the possibility that a derivative of $N R$ has been displaced from the GNP surface by 2-M E. The fluorescence intensity of this derivative is greater than that of $1 \mu \mathrm{M} \mathrm{NR}$ (used to prepare NRGNPs), which indicates that the quantum yield of the product is greater than that of NR. We note that the fluorescence (excitation and emission wavelengths and intensity) of NR does not change in the presence of $10 \mu \mathrm{M}$ 2-ME. Thus, it is our belief that the new product forms on the GNP surface. To further confirm our hypothesis, the solution of the product was concentrated and subjected to mass spectrometry, which resulted in a peak at $\mathrm{m} / \mathrm{z} 282$ that is different from that observed for $N R$ ( $\mathrm{m} / \mathrm{z}$ 318). The maximum excitation wavelength for the concentrated product is $\sim 480 \mathrm{~nm}$, which is also different from that of NR (580 $\mathrm{nm})$. To provide additional evidence to distinguish between the structures of the product and NR, we conducted $\mathrm{CE}$ separation at $\mathrm{pH}^{*}$ 1.9. At this low value of $\mathrm{pH}$, the electroosmotic flow was negligible, and thus, only positively charged solutes were detected. The migration time of the product (7.5 min) was longer than that of NR ( $4.7 \mathrm{~min})$, which indicates that there is less positive charge in the product [e.g., the lack of an $\mathrm{N}\left(\mathrm{C}_{2} \mathrm{H}_{5}\right)_{2}$ residue]. From these results, we tentatively suggest that the chemical formula for the product is $\mathrm{C}_{16} \mathrm{H}_{8} \mathrm{CINO}_{2}$; i.e., the product formed by substitution of the $\mathrm{N}\left(\mathrm{C}_{2} \mathrm{H}_{5}\right)_{2}$ residue in $\mathrm{NR}$ with a chloride ion from $\mathrm{HAuCl}_{4}$.

Effect of $\mathbf{p H}$. The $\mathrm{pH}$ of the solution might play a role in determining the sensitivity of our analysis because it affects such factors as the adsorption and desorption of NR and the product, the yield of the product, the quantum yields for NR and the product, and the displacement capability of thiols. To monitor the fluorescence changes related to NR and the product simultaneously, the excitation wavelength was set at $543 \mathrm{~nm}$. Table 1 indicates that the fluorescence of $\mathrm{NR}$ is insensitive to $\mathrm{pH}$ and to the presence of 2-ME (no reaction between 2-ME and NR). The weak fluorescence intensities of the solution at $\mathrm{pH} 4.0$ and 5.0 indicate that a slight desorption occurs of the NR product from the GNP surface. We note that the adsorption of NR on the GNP surface decreases slightly with decreasing $\mathrm{pH}$, while the fluorescence intensities at $610 \mathrm{~nm}$ of the solutions containing NRGNPs increases slightly upon decreasing the $\mathrm{pH}$ in the absence of 2-M E . It is also important to point out that the spectral profiles of the solution containing the NRGNPS and 2-ME are narrower, and the blue shift is more apparent at low $\mathrm{pH}$. In the presence of 2-M E, the fluorescence of the NRGNPs increased upon decreasing the $\mathrm{pH}$ in the range 4.0-9.0. This observation implies that at low 
values of $\mathrm{pH}$, the yield of the product was greater, the efficiency of the displacement of the product by 2-M E was higher, or both. To differentiate the individual effects, we dissolved the GN Ps by adding $20 \mathrm{mM} \mathrm{CN}^{-}$to the solutions. The greater fluorescence observed at low pH strongly suggests that the yield was higher under acidic conditions. Again, as in the case when 2-ME was added, the emission spectra are broad, and the maximum emission wavelengths were longer at high values of $\mathrm{pH}$, mainly because of overlap of the spectra corresponding to $N R\left(\lambda_{\mathrm{em}}=652 \mathrm{~nm}\right)$ and to its product $\left(\lambda_{\mathrm{em}}=610 \mathrm{~nm}\right.$ ). Our reasoning was supported by increases in fluorescence at 652 and $610 \mathrm{~nm}$ when we exited the solutions having higher $\mathrm{pH}(8.0$ and 9.0$)$ at $580 \mathrm{~nm}$ and the solutions having lower $\mathrm{pH}(6.0$ and 7.0$)$ at $480 \mathrm{~nm}$, respectively.

Relative to the results obtained upon adding $2-M E$, the fluorescence intensities are stronger following the addition of $\mathrm{CN}^{-}$, which suggests that the displacement efficiency of the product and NR by 2-M E from the GNP surface is another contributor to the fluorescence changes listed in Table 1 . At high $\mathrm{pH}$, only small amounts of NR are displaced by 2-ME, mainly as a result of Coulombic repulsion between citrate ions on the NRGN P surface and anionic 2-ME ( $\left.\mathrm{pK}_{\mathrm{a}}=9.6\right)$. At low pH, on one hand, the degrees of ionization of both citric acid and 2-M E are decreased, and thus, the force of their repulsion is weakened; once neutral 2-ME adsorbs onto the GNP surface through $\mathrm{Au}-\mathrm{S}$ bonding, the charge density of the GNPs decreases. On the other hand, the degree of protonation of $\mathrm{NR}$, which possesses two nitrogen atoms, is greater at low pH, and thus, its interactions with the GNP surface, which most likely occur through $\mathrm{Au}-\mathrm{N}$ interactions, and with citrate and $2-\mathrm{M} \mathrm{E}$ through hydrogen bonding are weakened..$^{40} \mathrm{As}$ a result, the displacement of both $\mathrm{NR}$ and the product by 2-ME are more effective at low values of $\mathrm{pH}$. With this phenomenon in mind, we believe that it is preferable to conduct experiments at $\mathrm{pH}$ 4.0. Under such a condition, the detection limit for 2-ME at a signalto-noise ratio $(\mathrm{S} / \mathrm{N})$ of 3 is $12.8 \mathrm{nM}$. At the concentration of $1 \mu \mathrm{M}$ of 2-ME, the reproducibility of this method is quite good (the relative standard deviation for the fluorescence intensity in five consecutive measurements is $2.1 \%$.

Adsorption and Desorption of NR. To optimize sensitivity, it is essential to control the concentration of $\mathrm{NR}$ used to equilibrate the GNPs. When using NR at a concentration $>3.0 \mu \mathrm{M}$, the GNPs aggregated at $\mathrm{pH} 4.0$, and free NR was found in the bulk solution, as indicated by increases in the fluorescence at $652 \mathrm{~nm}$. This phenomenon caused higher background signals and, thus, a deterioration of the system's sensitivity. From the inflection point of the plot of the fluorescence intensity at $652 \mathrm{~nm}$ vs the concentration of NR (not shown), we estimate that the saturated NR concentration is $0.95 \mu \mathrm{M}$, which corresponds to $3560 \mathrm{NR}$ molecules/GNP. Thus, $1.0 \mu \mathrm{M}$ NR was used to equilibrate the GNPs.

The fluorescence of NR at $652 \mathrm{~nm}$ is quenched dramatically in a short period of time ( $<10 \mathrm{~min}$ ) by the GNPs, which suggests that adsorption of NR on the GNPs occurs rapidly. We note that the fluorescence increased and a blue shift took place in the first $2 \mathrm{~h}$ of the equilibration, and then gradually decreased during the period between 2 and $6 \mathrm{~h}$. Thus, we believe that the product slightly desorbed, probably because of the coverage of NR molecules on the GNP surface. The fluorescence at $610 \mathrm{~nm}$

(40) Cser, A.; Nagy, K.; Biczók, L. Chem. Phys. Lett. 2002, 360, 473-478.

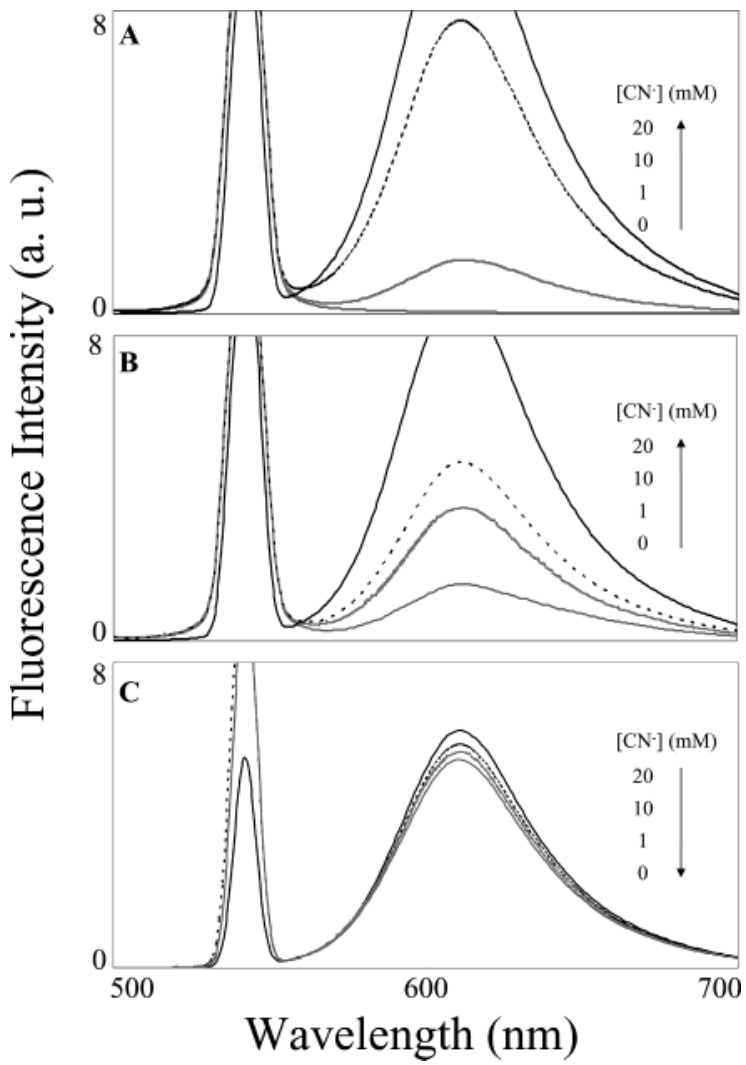

Figure 4. Effect of $\mathrm{CN}^{-}$on the emission spectra of solutions containing the NRGNPs: A, no 2-ME; B, $10 \mu \mathrm{M}$ 2-ME added to the solutions prior to the addition of $\mathrm{CN}^{-} ; \mathrm{C}, \mathrm{CN}^{-}$added to the supernatants after centrifugation of a solution containing NRGNPs and $10 \mu \mathrm{M}$ 2-ME. Other conditions are identical to those in Figure 3.

gradually increased upon increasing the reaction time and reached a plateau after $7 \mathrm{~h}$, which is a process that is much slower than the time required for $\mathrm{NR}$ adsorption to reach equilibrium. In terms of sensitivity, we suggest a reaction time of $12 \mathrm{~h}$ between $\mathrm{N} R$ and the 32-nm GNPs.

To further support our hypotheses that 2-ME is adsorbed on the GNP surface and that the GNPs have a quenching effect on the fluorescence of the solution, we added $\mathrm{CN}^{-}$separately to solutions of NRGNPs in the absence and presence of 2-M E. Figure $4 A$ and $B$ indicate that the fluorescence intensity at $610 \mathrm{~nm}$ (recorded $3 \mathrm{~min}$ after the addition of $\mathrm{CN}^{-}$) increases upon increasing the $\mathrm{CN}^{-}$concentration, with a slower reaction rate observed in the presence of 2-M E. The slow rate of dissolution is due mainly to the decreased surface area of the NRGNPs once they are aggregated and their surfaces have been passivated with 2-M E. ${ }^{41}$ Because smaller GN Ps that are more difficult to centrifuge may have formed as a result of the dissolution of large GN Ps in the presence of 2-M E, it is possible that the fluorescence intensity is quenched by the smaller GNPs in the supernatants. To test this possibility, we added $\mathrm{CN}^{-}$to the supernatants. The fluorescence intensity decreased slightly upon increasing the $\mathrm{CN}^{-}$ concentration, as presented in Figure $4 \mathrm{C}$, which indicates that the supernatants do not contain small GNPs.

Determination of Thiols. The results presented above indicate that it is possible to determine the concentrations of other

(41) Weisbecker, C. S.; M erritt, M. V.; Whitesides, G. M . Langmuir 1996, 12, 3763-3772. 
Table 2. Comparison of the Dynamic Range and LOD for Thiols Measured Using NRGNPs by Three Different Detection Modesa

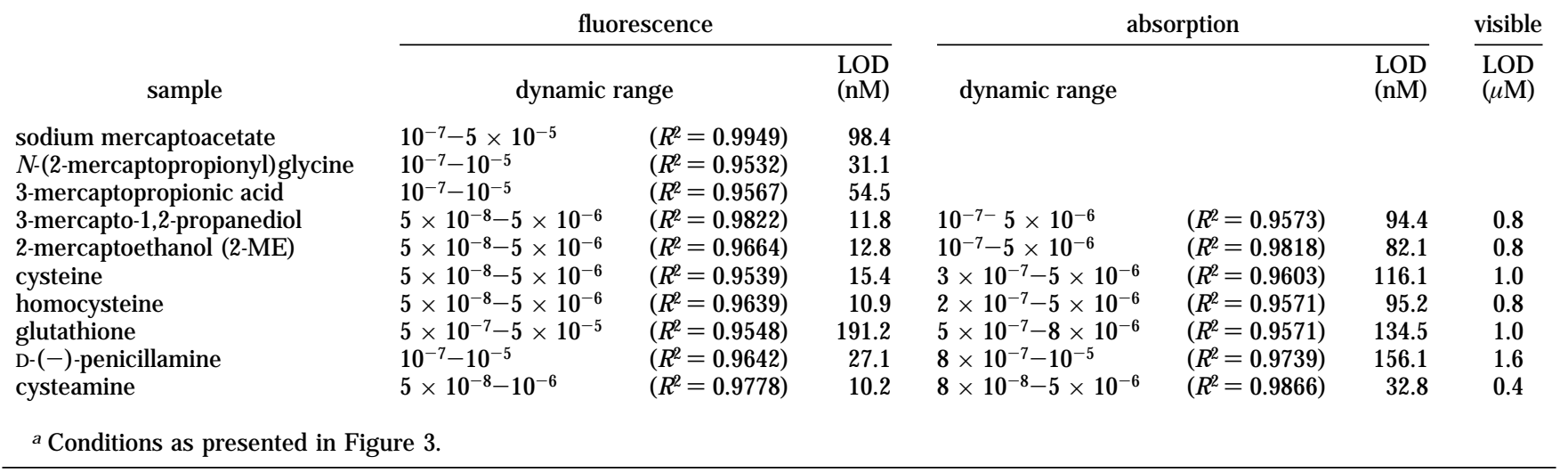

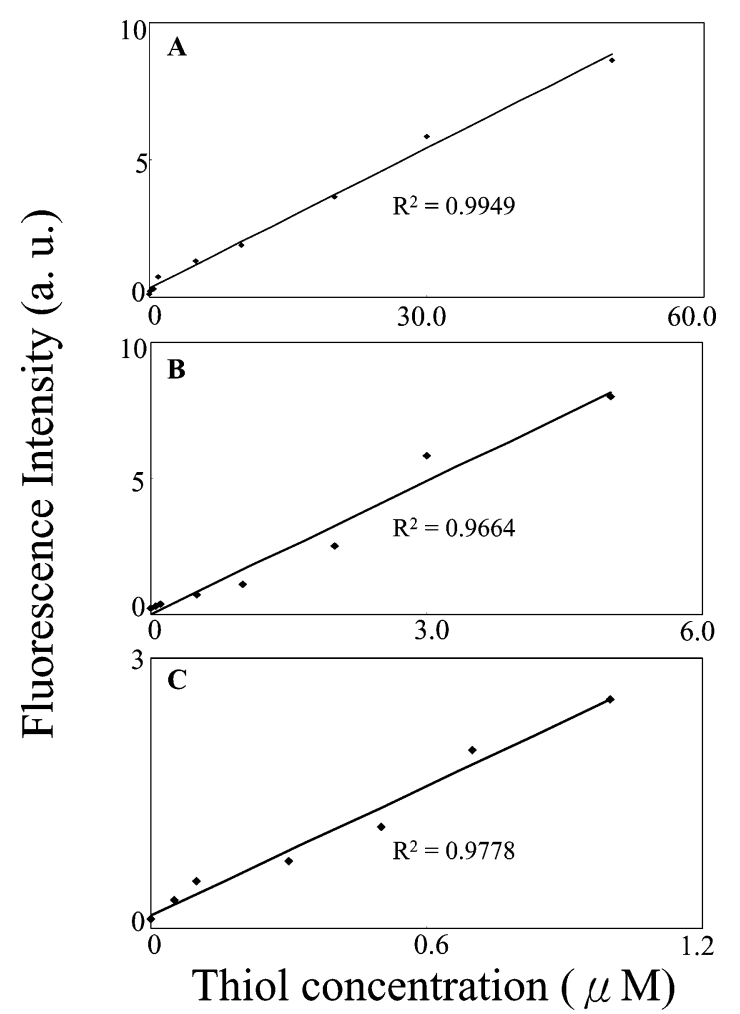

Figure 5. Linear plots of fluorescence intensity as a function of thiol concentration: A, sodium mercaptoacetate; B, 2-ME; C, cysteamine. Other conditions are identical to those described in Figure 3.

thiols by using NRGNPS. We tested a number of thiols, including some aminothiols. We plotted the fluorescence intensities ( $\lambda_{\mathrm{ex}}=$ $543 \mathrm{~nm}, \lambda_{\mathrm{em}}=610 \mathrm{~nm}$ ) of the solutions against the thiol concentrations. Three representative plots are presented in Figure 5; each one exhibits good linearity $\left(R^{2}>0.95\right)$. Table 2 indicates that the limits of detection (LOD) for 3-mercapto-1,2-propanediol, 2-ME, cysteamine, cysteine, and homocysteine at $\mathrm{S} / \mathrm{N}=3$ are $11.8,12.8,10.2,15.4$, and $10.9 \mathrm{nM}$, respectively. We note that the LODs are 5 times lower when the solutions are excited at 480 $\mathrm{nm}$. The LODs for cysteine and homocysteine are much lower than those in plasma $(\mu \mathrm{M})$, which suggests that this method has great potential for diagnostic purposes. It is interesting to note that the LODs are relatively higher for the thiols containing a carboxylate group, such as sodium mercaptoacetate $\left(\mathrm{pK}_{\mathrm{a}}=3.3\right)$, when compared to the neutral thiols, such as 2-ME. This phenomenon is due mainly to lower displacement efficiencies (weaker adsorptions) of the acidic thiols that possess a greater degree of negative charge density. The relatively poor sensitivity ( $L O D=191.2 \mathrm{nM}$ ) for glutathione (reduced form) is likely to be due to steric effects. It is important to point out that the disulfide compounds (e.g., oxidized glutathione) do not induce fluorescence increases.

To highlight the selectivity of this simple approach, we analyzed the presence of thiols separately at a concentration of $1.0 \mu \mathrm{M}$ in the presence of proteins $\left(10^{-5} \mathrm{M}\right)$, such as BSA and hemoglobin, and small organic compounds $\left(10^{-4} M\right)$, such as acetic acid, propylamine, and 2-propanol. The interference of those small compounds in the detection system is negligible, mainly because they interact much more weakly with the GNPs than do the thiols. Although we did see slight color changes (red shift) when adding the proteins, mainly due to a slight degree of aggregation, the recovery values for the analytes based on fluorescence measurements $(n=5)$ are $\sim 99$ to $102 \%$ The reason the proteins did not cause significant interference in the fluorescence intensity is because these proteins are much larger (several nanometers in diameter) when compared to the simpler thiols, and thus, the displacement of the product of NR from the GNP surface is quite inefficient. It has been shown that only several protein molecules adsorb onto each GNP, depending on their relative sizes. ${ }^{42,43}$ It is also possible that the small amounts of released product of $N R$ bind noncovalently to the BSA that adsorbs onto GNPs. ${ }^{44}$ Consequently, the fluorescence is quenched by the GNPs. It is also important to point out that the NRGNPs are quite stable in the presence of $10^{-5} \mathrm{M} \mathrm{BSA} \mathrm{(pl} \mathrm{4.5)} \mathrm{in} \mathrm{PBS} \mathrm{solution}$ ( $\mathrm{pH}$ 7.4), as supported by the TEM image (Figure 6A) or at 20 $\mathrm{mM}$ glycine ( $\mathrm{pH}$ 9.0) (TEM image is similar to Figure $6 \mathrm{~A}$ ); this stability is due mainly to Coulombic repulsion. ${ }^{45}$ This finding allows us to use NRGNP equilibrated with $10^{-5} \mathrm{M} \mathrm{BSA}$ at $\mathrm{pH} 9.0$

(42) Gole, A.; Dash, C.; Ramakrishnan, V.; Sainkar, S. R.; M andale, A. B.; Rao, M.; Sastry, M. Langmuir 2001, 17, 1674-1679.

(43) Cobbe, S.; Connolly, S.; Ryan, D.; Nagle, L.; Eritja, R.; Fitzmaurice, D. J. Phys. Chem. B 2003, 107, 470-477.

(44) Bertsch, M.; M ayburd, A. L.; Kassner, R. J. Anal. Biochem. 2003, 313, 187195.

(45) Huang, Y.-F.; Huang, C.-C.; Chang, H.-T. Langmuir 2003, 19, 7498-7502. 

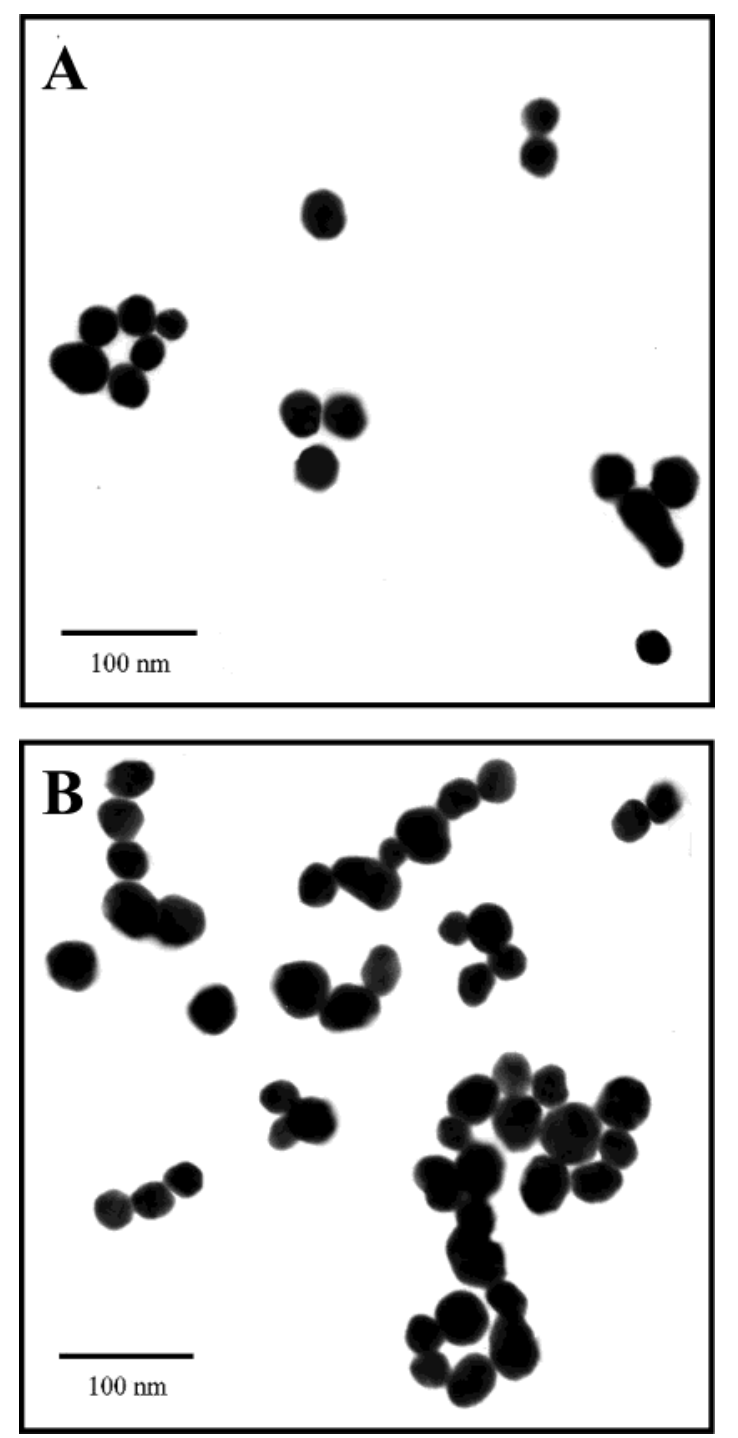

Figure 6. TEM images of NRGNPs in the presence of BSA. A and $\mathrm{B}$ : without and with 2-ME in $\mathrm{PBS}, \mathrm{pH} 7.4$, respectively. The concentrations for 2-ME and BSA are 1 and $10 \mu \mathrm{M}$, respectively.

to determine the presence of 2-ME in PBS, with a LOD of 13.5 $\mathrm{nM}$. The TEM image presented in Figure $6 \mathrm{~B}$ indicates that, in the presence of 2-ME, the NRGNPs that had been treated with BSA aggregate to some extent in PBS.

At $\mathrm{pH} 4.0$, once the neutral (e.g., 2-ME) and positively charged thiols (e.g., cysteamine) become adsorbed, they reduce the charge density of the GNPs and, thereby, induce their aggregation. ${ }^{41}$ As a consequence, the color changes from maroon to purple, and the UV-vis absorption undergoes a red shift ( $\lambda_{\max }$ shifted from 528 to $538 \mathrm{~nm}$ ) in the presence of neutral thiols that is a typical and interesting phenomenon for nanoparticles as they increase in size. The aggregation is obvious even in the presence of positively charged thiols, which cause the color to change to lavender. Figure 7 displays the different colors and TEM images of the NRGNPs after adding negatively charged, neutral, and positively charged thiols. Since the color change of NRGNPs in the presence of reduced glutathione is much slower (30 $\mathrm{min}$ ) than those (immediately) in the presence of cysteine and homocysteines, it is easy to discriminate glutathione from the other two thiols. It is also important to point out that oxidized glutathione
A. Negatively charged thiols
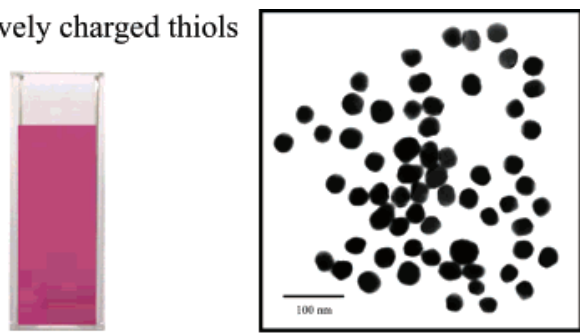

B. Neutral thiols
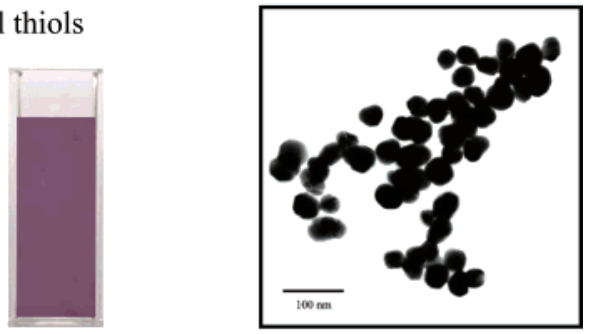

C. Positively charged thiols
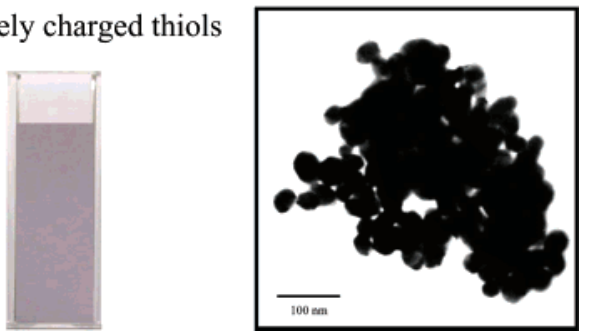

Figure 7. Colors and TEM images for NRGNPs in the presence of thiols at $\mathrm{pH}$ 4.0. Sodium mercaptoacetate, 2-ME, and cysteamine are representatives for negatively charged $(A)$, neutral $(B)$, and positively charged thiols $(C)$, respectively. The concentrations for the three representative thiols are $10 \mu \mathrm{M}$.

does not induce any color change within $3 \mathrm{~h}$. These results reveal that we can easily determine by the naked eye what types of thiols are present. Table 2 lists the color change of the NRGN Ps in the presence of neutral and positively charged thiols at concentrations $>1.0 \mu \mathrm{M}$ that are visible by the naked eye. As listed in Table 2, a colorimetric approach for the use of the NRGNPs in determining the presence of neutral (i.e., those lacking carboxylate groups) and positively charged thiols (i.e., those forming ammonium ions), with values of the LOD at the submicromolar level, by plotting the decrease in the ratios of the absorbance at $528 \mathrm{~nm}$ for neutral thiols and positively charged thiols against the thiol concentration. The linear dynamic range of the plot spans at least 1 order of magnitude $\left(R^{2}>0.95\right)$. Using NRGNP equilibrated with $B S A$, we can determine 2-ME in PBS (TEM image presented in Figure 6B) in a similar linear range, but with slightly higher LODs (e.g., 96.4 $n M$ for 2-ME). Again, such low values of the LOD suggest that the colorimetric approach is sensitive enough for determining the concentrations of cysteine and homocysteine in plasma and urine samples after separation.

\section{CONCLUSIONS}

We describe simple assays based on FRET and aggregation (colorimetric approach) for the selective determination of thiols using NRGNPs. The fluorescence of NRGNPs at $652 \mathrm{~nm}$ is suppressed because of FRET between NR and GNP, whereas the fluorescence at $610 \mathrm{~nm}$ is increased by the presence of thiols. The different optical (excitation, emission, and quantum yield) and mass spectral properties observed indicate that NR forms a 
new product upon interacting with the GNP surface. At pH 4.0, the increase in fluorescence at $610 \mathrm{~nm}$ is a result of the displacement of this product by the thiols, which is a process that allows the selective analysis of these thiols with LODs near 10 $\mathrm{nM}$. Although the sensitivity slightly decreases upon increasing the $\mathrm{pH}$, this method can be applied to determination of the presence of thiols to at least the submicromolar level at different values of $\mathrm{pH}$ (4.0-9.0). Since neutral and positively charged thiols induce aggregation of NRGNPs at pH 4.0 to different extents, but negatively charged thiols do not, colorimetric detection allows determination of the type of thiols at the submicromolar level. Depending on the evolution of color change, the NRGNPS allow one to discriminate glutathione from cysteine and homocysteine. When conducting a dual assay (fluorescence and colorimetric detection), the NRGNPs provide the capability of determining the type of thiols that are present. The results presented in this study indicate the diagnostic potential for NRGNPs in conjunction with enzyme assays for the analysis of traces of thiols in biological samples. We also highlight that NRGNPs in the presence of BSA are quite stable and are useful for determining the presence of thiols in PBS. Our future goals include using NRGNPs for sensing thiols in biological samples that are separated by capillary or microchip electrophoresis.

\section{ACKNOWLEDGMENT}

This work was supported by the National Science Council of Taiwan, the Republic of China, under Contract N o. NSC 92-2113M -002-051. The authors gratefully thank Professor J en-Taie Shiea, Dr. Jing-Yueh J eng, and $M$ iss Yu-Ching Cheng ( $N$ ational Sun $Y$ atSen University, Kaohsiung, Taiwan) for the mass spectroscopic measurements.

Received for review February 7, 2004. Accepted April 9, 2004.

AC049787S 